\title{
Integration of Eco-Friendly POF Based Splitter and Optical Filter for Low-Cost WDM Network Solutions
}

\author{
Mohammad Syuhaimi Ab-Rahman, Hadi Guna, \\ Mohd Hazwan Harun, Latifah Supian and Kasmiran Jumari \\ Universiti Kebangsaan Malaysia, Selangor Darul Ehsan \\ Malaysia
}

\section{Introduction}

The field of 'green technology' encompasses a continuously evolving group of methods and materials, from techniques for generating energy to non-toxic cleaning products. The present expectation is that this field will bring innovation and changes in daily life of similar magnitude to the 'information technology' explosion over the last two decades. In these early stages, it is impossible to predict what 'green technology' may eventually encompass (Gupta and Khurana 2010).

Nowadays, the whole world of telecommunications and information communities is facing a more and more serious challenge, namely on one side the transmitted multimedia-rich data are exploding at an astonishing speed and on the other side the total energy consumption by the communication and networking devices and the relevant global $\mathrm{CO}_{2}$ emission are increasing terribly. It has been pointed out that 'currently $3 \%$ of the worldwide energy is consumed by the information \& communications technology (ICT) infrastructure that causes about $2 \%$ of the world-wide $\mathrm{CO}_{2}$ emissions, which is comparable to the world-wide $\mathrm{CO}_{2}$ emissions by airplanes or one quarter of the world-wide $\mathrm{CO}_{2}$ emissions by cars' (Janota and Hrbček 2011).

According to the recent research report of Ericsson Media Relations, energy costs account for as much as half of a mobile operator's operating expenses. Therefore, telecommunications applications can have a direct, tangible impact on lowering greenhouse gas emissions, power consumption, and achieve efficient recycling of equipment waste. Moreover, to find radio networking solutions that can greatly improve energy-efficiency as well as resource-efficiency is not only benefit for the global environment but also makes commercial sense for telecommunication operators supporting sustainable and profitable business. Within the framework of 'green communications', a number of paradigm-shifting technical approaches can be expected, including but not limited to energy-efficient network architecture \& protocols, energy-efficient wireless transmission techniques (e.g., reduced transmission power \& reduced radiation), cross-layer optimization methods, and opportunistic spectrum sharing without causing harmful interference pollution (Ericsson 2008). 
The green technology wavelength division multiplexing based on polymer optical fiber (GTWDM-POF) network solution is presented. Green technology polymer optical fiber (GTPOF) splitter has been fabricated by environmental friendly fusion technique, as an effective transmission media to split and recombine a number of different wavelengths. Two different wavelengths from ecologically friendly light emitting diode (LED) were fully utilized to transmit two different sources of systems; Ethernet connection and video transmission system. Red LED which in $650 \mathrm{~nm}$ wavelength capable to download and upload data through Ethernet cable while green LED in 520nm wavelength transmits a video signal. Special filter has been placed between the splitter and receiver-end to ensure GT-WDM-POF network system can select and generate a single signal as desired. Efficiency of both devices and network were observed. The material, fabrication method, system \& application approach in this chapter are based on the environment friendly solution to reduce the power consumption \& wastage without affecting the system performance. Our GT-POF splitter and GT-WDM-POF network solution proposed in this paper are the first reported up to this time.

\subsection{Eco-friendly sources}

LEDs, or light emitting diodes, are the light source in solar powered products. This solidstate product is composed of a semiconductor diode. A semiconductor is a material that can conduct electricity. A semiconductor diode is composed of a semi-conductive crystal that has added impurities in order to create a positive and negative side; since current flows in one direction through the diode. A region is then created in between the positive and negative zones, called a $P N$-junction, which is where the action takes place within the diode; in our case it emits light. LEDs are preferably used due to the many advantages can be offered: Efficiency: An incandescent light requires much more energy to properly heat the filament in order to generate light. The light produced by an LED is a cool light. More light is produced per watt in an LED than an incandescent. Even more energy can be saved if the light is solar powered.

LED bulbs are widely used in visible light communication which replacing Wi-Fi and offer much higher capacity. Color: LEDs do not require filters, like colored bulbs, in order to create a specific colored light. Color is produced based on the material of the semiconductor. The different color represents the frequency of carrier signal that can be used to increase the data capacity by introducing Wavelength Division Multiplexing (WDM). Size: LEDs come in many different sizes since they are not constrained to creating a vacuum in which to house the filament. LEDs can be smaller than $2 \mathrm{~mm}$. On/Off Time: An LED takes only microseconds to achieve its full brightness. This is ideal in a solar powered light that is running off of a battery that has determinate energy life. On/Off time or blinking of Led also represent the capacity of data that can be modulated. Cycling: In applications that are cycled between on and off frequently, like an outdoor solar light, LEDs are ideal since they won't burn out quickly. Lifetime: The lifetime of the LED greatly exceeds its incandescent, and even its fluorescent counterparts. An average lifetime of an incandescent light is 1,000-2,000 hours and a fluorescent bulb is 10,000-15,000 hours. The LED, on the other hand, has a typical lifetime of 35,000-50,000 hours. Light Dispersement: An LED is designed to focus its light, so where an incandescent or fluorescent may seem brighter since the light radiates in all directions, the LED light can be directed to a specific location without the use of an external reflector. 
The smaller beam size enable the light be injected to the multimode fiber for communication application. Ecologically Friendly: LEDs are more efficient than others, as stated above; so they conserve electricity, especially if they are solar powered. LEDs do not contain toxic chemicals like fluorescent bulbs do. Several incandescent and fluorescent bulbs will be used during the lifetime of a single LED. If your desire is to light a space and save the environment, then the clear choice is the LED. Solar powered LEDs are an additional benefit in that they require no additional energy costs.

Three major benefits of this solid-state lighting technology, shown in Fig. 1, can be summarized as follows: firstly, the inherent capability of solid-state sources to generate light with high efficiency is resulting in giant energy savings. Secondly, potentially huge environmental benefits are a result of the efficiency and durability of solid-state emitters, particularly light-emitting diodes based on inorganic semiconductors. Thirdly, solid-state emitters allow one to control the emission properties with much greater precision, thereby allowing one to custom-tailor the emission properties for specific applications (Schubert, Kim et al. 2006).

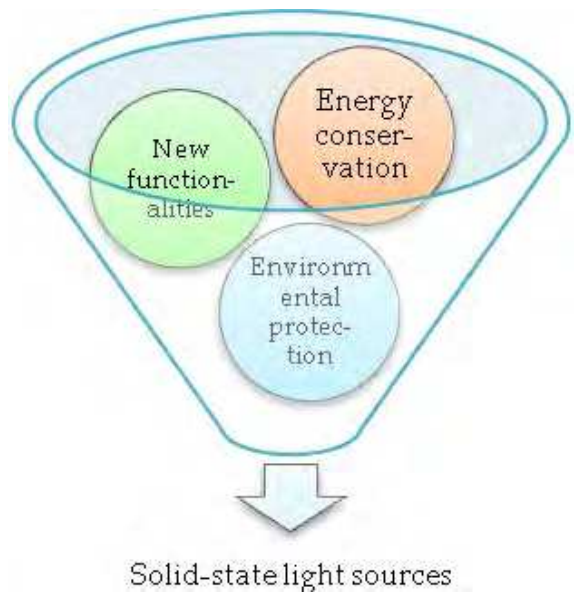

Fig. 1. Benefits enabled by solid-state light sources

\subsection{Green technology medium}

Nowadays, polymer optical fiber (POF) become alternative transmission media replacing copper or even glass fiber for short-haul communication. POF links are becoming increasingly popular for applications such as computer or peripheral connections, control and monitoring, board interconnects and even domestic Wi-Fi systems. Unlike glass fiber, POF remains flexible while having a large diameter core and high numerical aperture (Keene and Selli 1989), lead to high capacity they can bring along the fiber. Moreover, the fiber is easy to handle with the potential for constructing networks using simple conductor and easy installation procedures while retaining some of the advantages of optical fiber such as electromagnetic interference (EMI) immunity, non-conducting cable, small size and security. Another feature is the use of visible light to transmit information (Kuzyk 2007). 
Due to wide advantages of POF over copper or even glass fiber, POF are used widely in various optical networks. Recent communication system over POF desires increasingly more bandwidth and therefore the wavelength division multiplexing (WDM) system is the solution that allows the transmission of information over more than just a single wavelength (color) and thus greatly increases the POF's bandwidth. WDM is a technique that multiple signals are carried together as separate wavelengths (color) of light in a multiplexed signal (Kuzyk 2007; Grzemba 2008; Ziemann, Zamzow et al. 2008).

\subsection{Multipurpose applications}

Video Communications efficiently utilizes energy, reduces unnecessary travel, and significantly shrinks carbon emissions. Video Communication allows our organization to reduce carbon footprint, since one of the most effective ways to reduce carbon dioxide emissions is to reduce unnecessary travel. It provides measureable result to help us achieve more with less. Video Communication is the green way to communicate. Through the introduction of Video Conferencing your activities can; a) communicate better and optimize work-life-balance, b) become more environmentally responsible and c) reduce the organization carbon footprint. The International environmental organizations specify video communications as an effective green-technology to reduce carbon footprint, thereby reducing global warming effects. The Nature Conservancy lists video conferencing as one of its 'Easy Things You can Do to Help Our Climate.' The World Wildlife Fund has released reports that demonstrate how video conferencing dramatically reduces carbon emissions.

Communication using video conferencing technology offer an ideal solution to enable us to reduce the amount of time you spend in your car or travelling, while allowing us to visually connected to anyone, anywhere, at any time. It improves the environment with lower hydrocarbon emissions, and a reduction of fuel consumption and pollution.

\subsection{Proposed network}

In wavelength division multiplexing based on polymer optical fiber (WDM-POF) system, many transmitters with different lights color to carry single information. For example, red light with 650nm wavelength modulated with Ethernet signal while blue, green, and yellow lights carry image information, radio frequency (RF), and television signal, respectively (Ericsson 2008). As shown is Fig. 2, Wavelength Division Multiplexer is the first passive device required in WDM-POF system and it functions to combines optical signals from multiple different single-wavelength end devices onto a single fiber.

Conceptually, the same device can also perform the reverse process with the same WDM techniques, in which the data stream with multiple wavelengths decomposed into multiple single wavelength data streams. The reverse process is called as de-multiplexing.

In general, POF splitter Conceptually, POF splitter has similar function, operates to couple or combine several optical data pulse as a single coupled signal. Hence, the development of wavelength division multiplexer based on POF splitter is possible.

Typically, the commercial POF splitter that manufactured commercially by some manufacturer priced expensively at approximately more than US\$250 in global market. There have been many techniques of fabricating POF splitter. These techniques include 


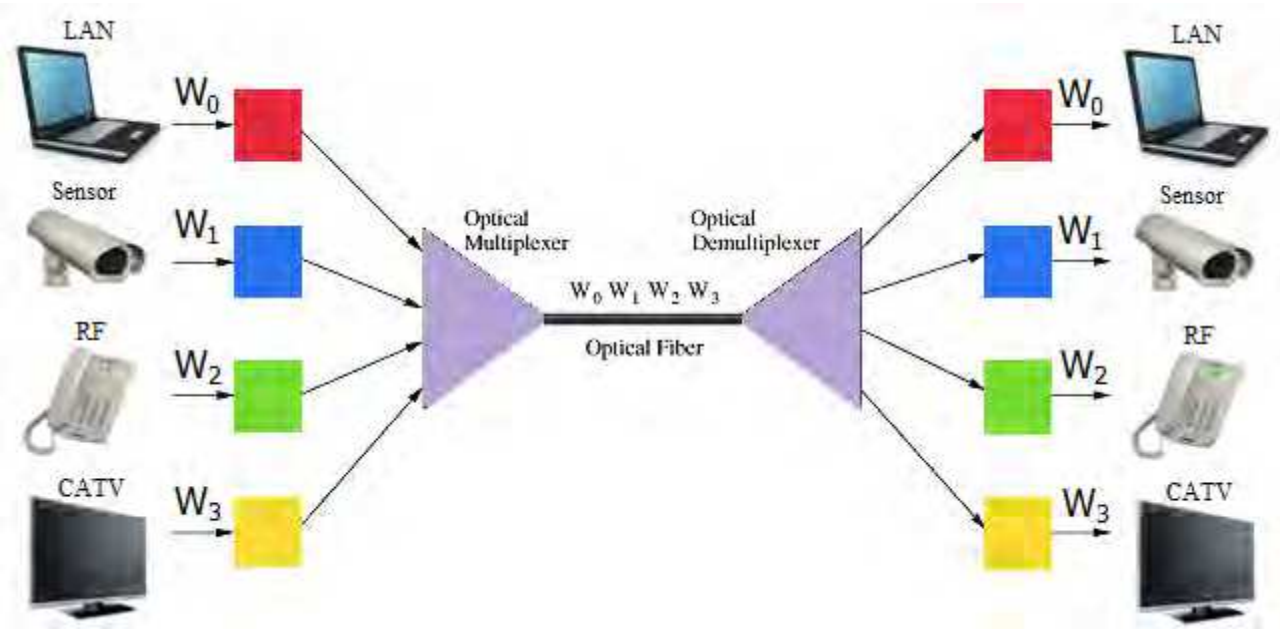

Fig. 2. Wavelength Division Multiplex (WDM) with an optical multiplexer and demultiplexer

twisting and fusion, side polishing, chemical etching, cutting and gluing, thermal deformation, moulding, biconical body and reflective body (Ehsan, Shaari et al. 2009).

For this chapter, fusion technique is practically proposed to fabricate POF splitter. Essentially, the term of 'fusion' defines the act or procedure of liquefying or melting by the application of heat (Imoto, Maeda et al. 1987). In order to develop the economical POF splitter, this study is undertaken to modify the typical fusion technique, whereby the technique is fully implemented by handwork. The heating elements and immune-to-heat tube (from the previous fusion technique) are changed in terms of availability and the appropriate twisting and pulling strengths are tuned specifically for the modified fusion technique (Kelly, May et al. 1995; Ab-Rahman, Guna et al. 2008). In this chapter, the characterization of the GT-POF splitter is carried out in order to determine the performance of device. Besides, study on how far the WDM-POF system can go, and how far color filter influences the output power of the system also reported.

\section{The WDM technology}

Three major benefits of this solid-state lighting technology can be summarized as follows: firstly, the inherent capability of solid-state sources to generate light with high efficiency is resulting in giant energy savings. Secondly, potentially huge environmental benefits are a result of the efficiency and durability of solid-state emitters, particularly light-emitting diodes based on inorganic semiconductors. Thirdly, solid-state emitters allow one to control the emission properties with much greater precision, thereby allowing one to custom-tailor the emission properties for specific applications (Schubert, Kim et al. 2006).

In wavelength division multiplexing based on polymer optical fiber (WDM-POF) system, many transmitters with different lights color to carry single information. For example, red light with 650nm wavelength modulated with Ethernet signal while blue, green, and yellow lights carry image information, radio frequency $(\mathrm{RF})$, and television signal, respectively 
(Ericsson 2008). WDM is the first passive device required in WDM-POF system and it functions to combines optical signals from multiple different single-wavelength end devices onto a single fiber. Conceptually, the same device can also perform the reverse process with the same WDM techniques, in which the data stream with multiple wavelengths decomposed into multiple single wavelength data streams. The reverse process is called as de-multiplexing. As we know, the most essential devices needed in common WDM-POF technology are transmitter, multiplexer, demultiplexer and receiver.

\section{Environmental friendly fabrication method}

For this chapter, fusion technique is practically applied to fabricate POF splitter. Essentially, the term of 'fusion' defines the act or procedure of liquefying or melting by the application of heat (Imoto, Maeda et al. 1987). In order to develop the economical POF splitter, this chapter is undertaken to modify the typical fusion technique, whereby the technique is fully implemented by handwork.

The heating elements and immune-to-heat tube (from the previous fusion technique) are changed in terms of availability and the appropriate twisting and pulling strengths are tuned specifically for the modified fusion technique (Kelly, May et al. 1995; Ab-Rahman, Guna et al. 2008). In this chapter, the characterization of the GT-POF splitter is carried out in order to determine the performance of device. Besides, chapter on how far the WDM-POF system can go, and how far color filter influences the output power of the system also reported.

The $1 \times N$ GT-POF splitter is an optical device, which ended by $N$ number of POF ports, while the other side ended by one POF port. Like other typical splitter, it is also possible to work bidirectional, whereby it works from the $N$ ports into 1 port (for coupling signal purpose), or vice versa (for splitting signals purpose). As an example, $1 \times 4$ GT-POF splitter developed by the jointing of four Polymethylmethacrylate (PMMA) POFs. Each inputs and output is connected with POF connecter as shown in Fig. 3.

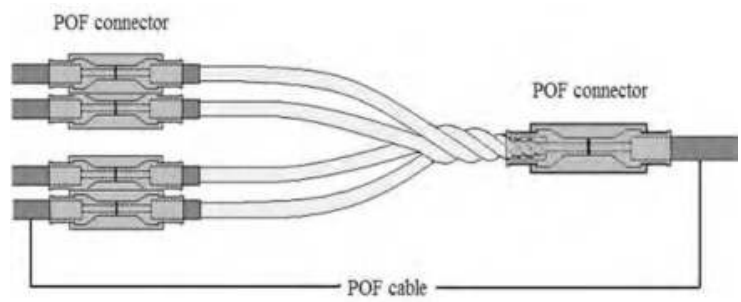

Fig. 3. The design of the $1 \times 4$ GT-POF splitter

For the filter design which able to eliminate unwanted signal and select the wavelength of the system as desired as shown in Fig. 4.

In development process of $1 \times N$ GT-POF splitter based on POF technology, multimode SIPOF type made of PMMA $1 \mathrm{~mm}$ core size fully utilized in this paper, as PMMA is one of the most commonly used optical materials, Due to its intrinsic absorption loss mainly contributed by carbon-hydrogen stretching vibration in PMMA core POF (Kuzyk 2007). 


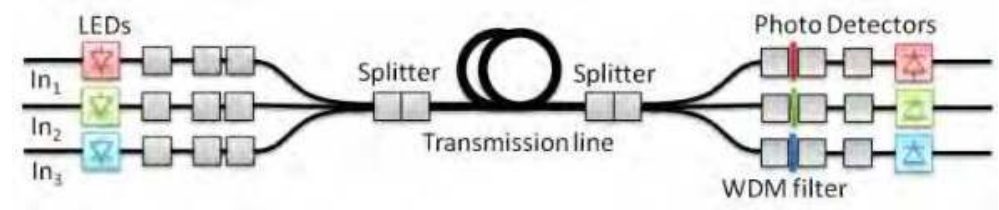

Fig. 4. GT-WDM-POF network architecture using $1 \times 3$ GT-POF splitter and color filters. The splitter can also be used as multiplexer and the splitter-filter combination can be performed as demultiplexer

To fabricate the final product of optical $1 \times N$ GT-POF splitter, some stages has to be done, start from fiber fusion, bundle formation and finalized with cable jointing. Fabricated through fusion method by fuses and combine $N$ number POFs (in bundle arrangement) and fabricate it ends part in a shape of fused-taper-twisted fibers (diameter $1 \mathrm{~mm}$ ). POFs will be twisted and pulled down while it is fused in a heat of flame. Heating process was done indirectly, while POFs covered by metal tube. Thus, heat was provided for POFs through metal tube heating.

For characterization process, here we choose a number of samples of $1 \times 4$ GT-POF splitter to measure the efficiency of the GT-POF splitter. The developed splitter must be able to properly coupling an optical signal to generate a single coupled signal efficiently, with low power loss. Optical power meter has been used to measure the optical power from POFs.

Bidirectional optical loss measurement is carried out in order to determine either side of the $4 \times 4$ GT-POF splitter with lower optical loss as final product of $1 \times 4$ GT-POF splitter before cutting the middle of the $4 \times 4$ GT-POF splitter. Red LED injected through each of inputs individually and separately from the right side (lights propagate leftward) in order to measures output powers and calculates the optical loss. Then, similar procedure is repeated for rightward measurement. Finally, optical loss for fused bundle in both directions analytically compared. The procedure explained above visualized as in Fig. 5.

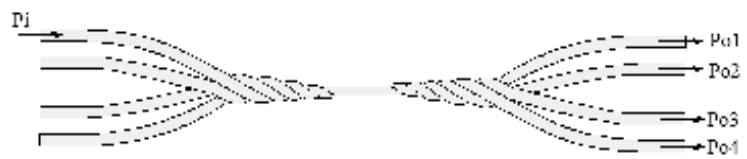

(a)

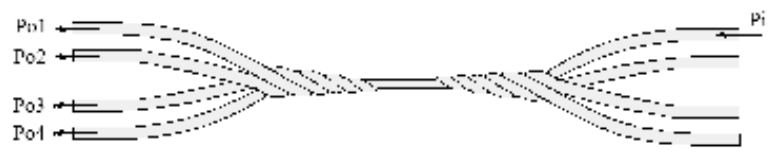

(b)

Fig. 5. Measurement process for bi-directional $4 \times 4$ GT-POF splitter

In order to measure the power efficiency of splitter, at first, red LED injected from transmitter pit into single POF cable ( $1 \mathrm{~mm}$ of core diameter) and obtained power defined as input power while output power obtained by injecting LED into POF splitter (through 
single POF port) and each POF ports (on cascaded side) measured by optical power meter. The procedure of input/output power measurement depicted in Fig. 6.

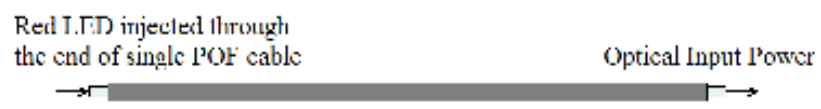

(a)

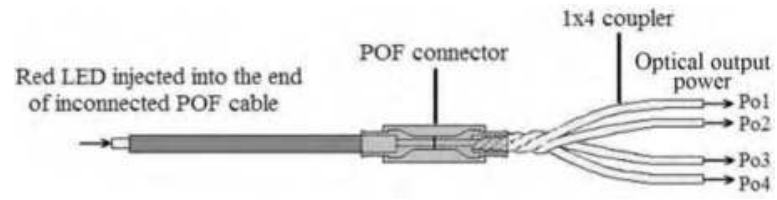

(b)

Fig. 6. The illustration of (a) input and (b) output power measurement

\section{Results}

In order to investigate precisely the exact value of power intensity for each POF outputs of fused bundle, bidirectional optical loss measurement has been carried out whereby injecting red LED through each of POF inputs on both sides of fused bundle separately. The average optical loss for fused POF bundle has been yet calculated for both directions (leftward and rightward) and then analytically compared. According to the observation above, it is revealed that the optical loss for fused bundle in different direction was not identical. Analytically, fused POF bundle has lower optical loss in rightward direction. Thus, right side of fused POF bundle selected as POF splitter because it might couple multiple optical signals and produce single optical signal with lower attenuation and higher efficiency compared to the other side. Indeed, optical loss for fused bundle mainly caused by physical changes on POF especially on fused taper twisted in which POFs in bundle arrangement were all fused, twisted and merged.

Through modified fusion technique (see Fig. 7), the ideal sample of fused-taper-twisted POFs successfully produced which the diameter of fused taper-twisted POF approaching $1 \mathrm{~mm}$. As the result of injecting red LED through the fused POF bundle, it is observed that red light pass smoothly through fused taper-twisted POFs. It is observed that all POF outputs did illuminate red light individually with different intensity power. So this means that no deformation occurred along fused taper-twisted POFs and this sample has ability of signal coupling.

For commercial purpose, research also produces a GT-POF splitter come out with a proper housing with concept of '1Malaysia' (called one Malaysia) as shown in Fig. 8, the housing wrapped by a beautiful Malaysian's attire sticker to promote the specialty of this local product. The efficiency of the signal transmitted by this splitter can be seen in Fig. 9.

By utilizing this GT-POF splitter, research also conduct a second project called GT-WDMPOF network by integrating a color filter inside the POF connection as shown in Fig.10. A certain information or data are carried by the transmitters where each transmitter carries signals of different wavelengths specified by the LED. The specialized designed color films are used to filter out any other wavelength that is not within the range. It will only allow one wavelength to get through the film and thus conveyed the data carried at the receiver. 


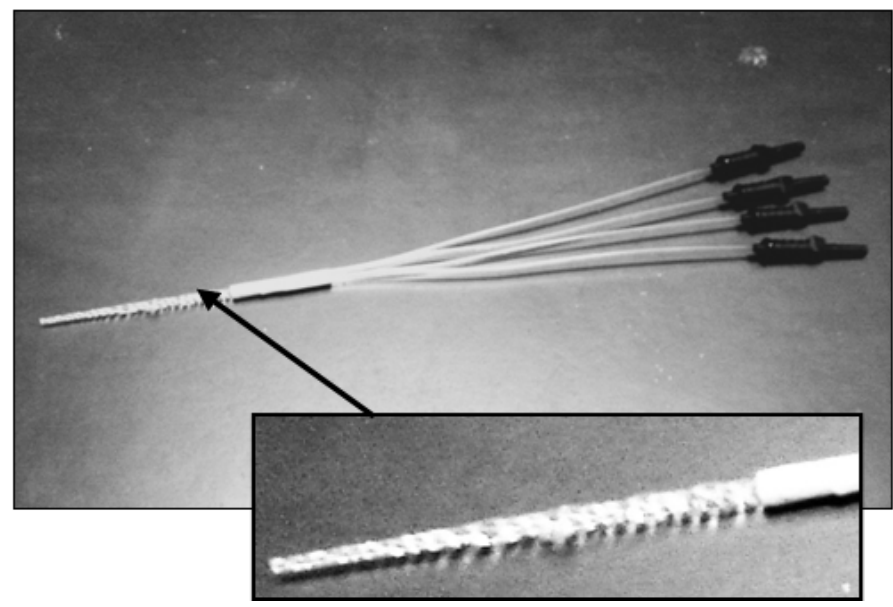

Fig. 7. Prototype of the novel $1 \times$ N GT-POF splitter; zoomed in picture visualize the fusedtaper-twisted POF

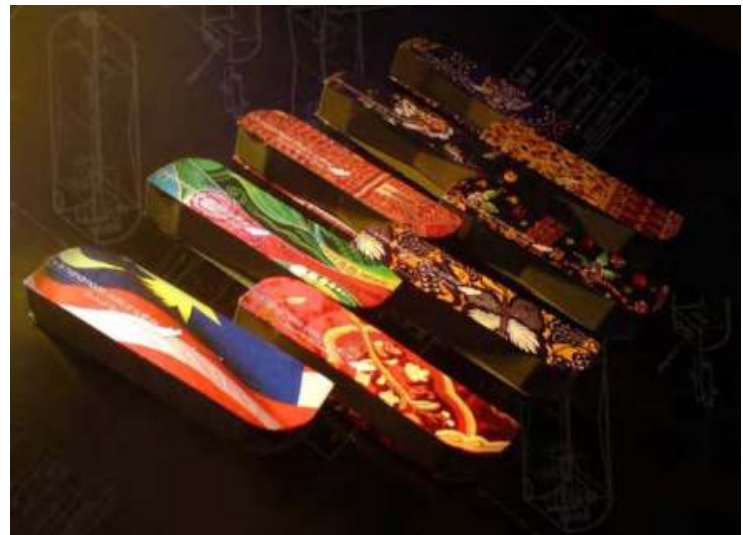

Fig. 8. Packed Prototype of the $1 \times$ N POF Splitter \& Demultiplexer called 1Malaysia ${ }^{T M}{ }^{\text {splitter }}$

Back to the GT-POF splitter, the fused taper-twisted part (refer to Fig. 7), where every four POFs were fused or combined becoming as so-called single POF, play major role in coupling four individual optical signals. The fused taper-twisted POFs should be fabricated as well as all fibers in bundle arrangement fused (combine one another via heat exposure) completely. Otherwise, the POF splitter would probably fail to work according to its main role, coupling the numbers of individual optical signal (Ab-Rahman, Guna et al. 2009; Ab-Rahman, Guna et al. 2009; Ab-Rahman, Guna et al. 2009).

The error could be occurred on it either while fabrication process or characterization test stages imposed on them. Irregularities of controlled heat while heating process exposed on the POFs become one of the major problem (Ab-Rahman, Guna et al. 2008; Ab-Rahman, Guna et al. 2009; Ab-Rahman, Harun et al. 2009), due to it lower melting point makes core structure of POF could be more sensitive on heating process. Once it is damaged, it is hard 


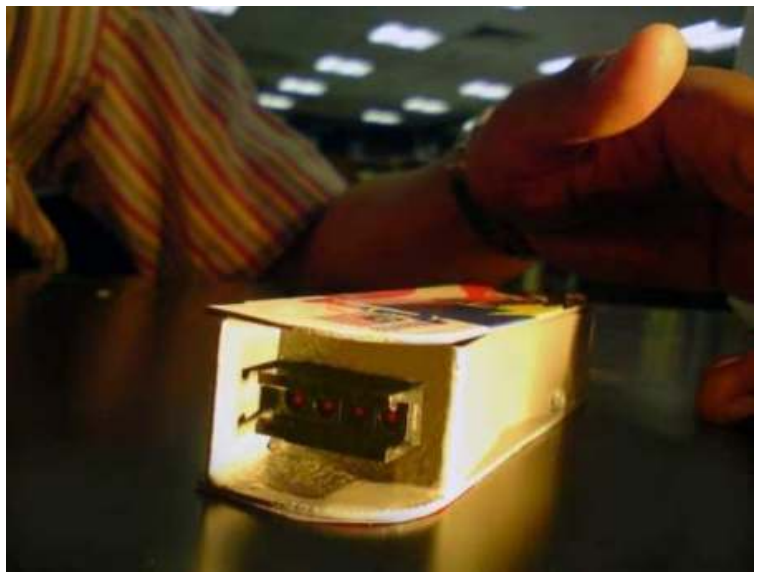

Fig. 9. Prototype of $1 \times N$ GT-POF splitter. The input signal is split into four channels which are suitable to distribute the application equally to the number of destination
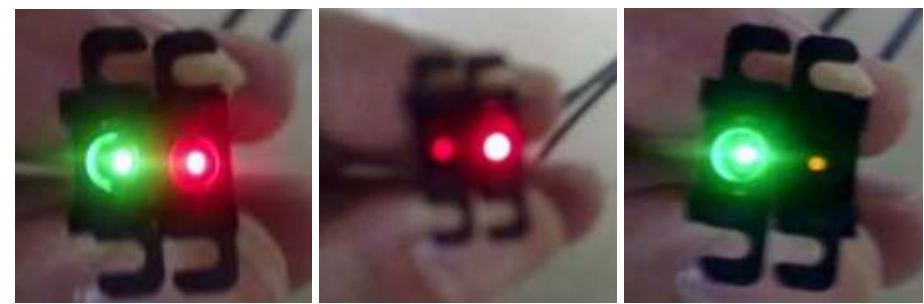

Fig. 10. Prototype of the $1 \times N$ POF Demultiplexer. The device is used to split the signal to different frequency (color). The multiplexed signal is separated according to the application (data \& video signal) respectively

to let a light pass through the core, or even not pass at all. It is so important to stop twisting and pulling POFs while the POF was getting frozen in order to prevent micro-scaled crack on core. That is why we use the metal tube while we conduct the indirect heating to fiber, it is to reduce this kind of damaged.

Furthermore, comparing with Imoto (1987) works, this fabrication method are produce less harmful gases such as nitrogen, sulfur or Carbon monoxide as produced by reaction of burning process using oxyhydrogen burner. The change of original diameter of POF considerably led to the change on optical properties including numerical aperture and maximum acceptance angle. All of these changes spoil light propagation principle based on total reflection; there would be much more rays of light refracted and propagate beyond cladding to atmosphere (Held 2002).

Indirect heating was used to minimize the undesirable deformation in the fused fiber bundle. This allows us easier fabrication and accurate control of the biconical taper. Furthermore, the continuous processing capability leads us to the reduction in fabrication time and improved yield. This method is expected to drastically reduce coupler fabrication costs (Imoto, Maeda et al. 1987). 
The change of original diameter of POF considerably led to the change on optical properties including numerical aperture and maximum acceptance angle. All of these changes spoil light propagation principle based on total reflection; there would be much more rays of light refracted and propagate beyond cladding to atmosphere (Held 2002).

After characterization had been done, if the LED injected directly through single POF cable, the obtained input power was $12 \mu \mathrm{W}$. As expected, output will be obtained minimum is 3 $\mu \mathrm{W}$ (as the power expected to be divided into 4 POFs equally). From the observation of power output measurement, the final result was obtained as shown in Fig. 11.
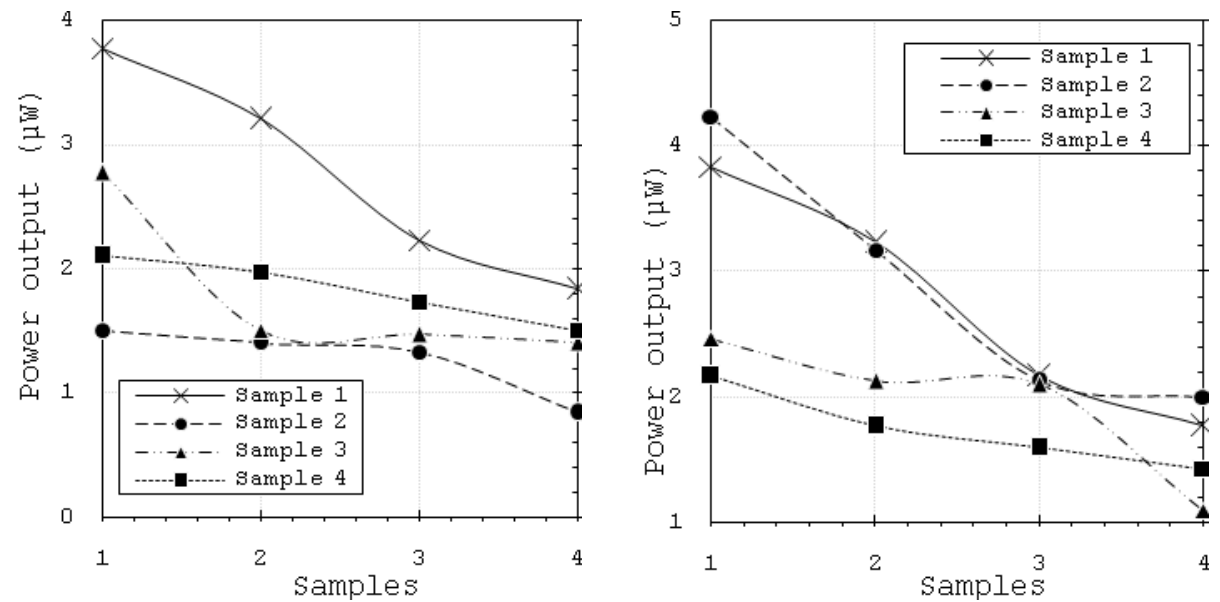

Fig. 11. The analysis of optical loss for fused bundle in both directions

Furthermore, it is stated that splitting ratio of the developed $1 \times N$ GT-POF splitter was not totally homogenous. In GT-WDM-POF network, the homogeneity concerned as essential criteria for POF splitter as optical multiplexer in coupling multiple optical signals. For example, if red, blue, green, and yellow lights injected through Port 1, Port 2, Port 3 and Port 4 , respectively, the blue light with $470 \mathrm{~nm}$ wavelengths will experience the greatest optical loss after all different wavelengths multiplexed. This would result in much more power dissipation in de-multiplexing mixed wavelengths into single blue wavelength whereby the optical power output of de-composed blue wavelength would be far less.

These happened because all POFs in bundle arrangement were not fused completely. Physically, the twisted-effect still could be seen on fused POFs and this indicates that all POFs not fully fused. In other word, the fused-taper-twisted POF was not formed well. Since the fusion technique was done by handwork, we found hard to fabricate and ensure all POF were fully fused. Obtained data was manipulated to determine the attenuation of each POF outputs as depicted in Fig. 12.

From analytical observation above, it is indicated that attenuation of each output is $0.25 \mathrm{~dB}$, $2.93 \mathrm{~dB},-0.63 \mathrm{~dB}$ and $-0.14 \mathrm{~dB}$, respectively. Different values show that each POF output has different characteristic of attenuation. After calculating, the minimum insertion loss for prototype is $2.41 \mathrm{~dB}$. We found that standard $1 \times N$ GT-POF splitter has $2.1 \mathrm{~dB}$ of minimum 


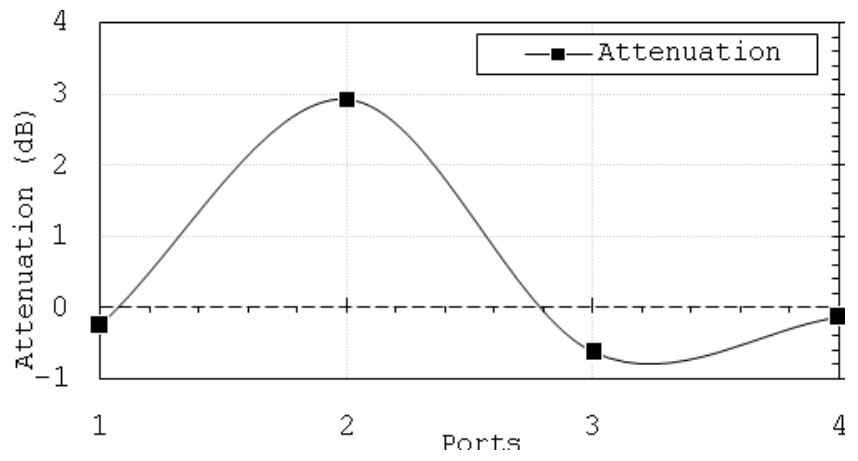

Fig. 12. Different attenuation on each POF output

insertion loss. So, the deviation of insertion loss between our novel and commercial splitter is not so large.

Comparison of hand-made and commercial splitter have been observed, in term of market price. Overall price for $1 \times 4$ GT-POF splitter cost is less than US $\$ 3$, but for the commercial one which available in market is cost not less than US\$250. Nowadays, many technology have been provided to coupling a signal, Low Cost $1 \times 2$ Acrylic-based Plastic Optical Fiber Coupler (Ehsan, Shaari et al. 2009) for example, but knowing that the fabrication techniques was very complicated, not to mention about their massive equipment needed, here GT-POF splitter can be seen as one of the most promising solution to face this kind of problem.

In this chapter, the optical loss is categorized as extrinsic loss due to the physical change of POF, LED projection to POF and the core-to-core connection and (Appajaiah, Wachtendorf et al. 2007; Kuzyk 2007). It is learned that the physical change of POF caused by fabrication process, where by diameter of POFs increasingly decrease to approach $1 \mathrm{~mm}$ and the POFs finally has taper-twisted shape. In characterization process, optical loss may present through the direct LED projection to POF surface. Besides, optical loss may also present through the connection between the fused taper-twisted POFs and POF cable (Appajaiah, Wachtendorf et al. 2007). The other aspect that playing an important role to transmit two different signal represented by different color on transmitter devices is the filter which is placed between the GT-POF splitter and the receiver section. In this research, two different LED was utilized; red LED $(650 \mathrm{~nm})$ transmit an internet line through LAN connection and green LED $(520 \mathrm{~nm})$ to deliver a high quality video signal to be displayed on a monitor screen. Analysis on the effectiveness of the filter itself also carried out. Here the comparison result of the efficiency of both green and red LED on their way to deliver a different signal to be split by GT-POF splitter, and optical power meter was placed in the output port right before the receiver port, as shown in Fig. 13.

The deviation between both signals was reach $3 \mathrm{~dB}$, while video transmission system showed a better quality of transmission system in low-cost WDM-POF system. The image quality of the video through WDM-POF method can be seen in Fig. 14.

Comparison for the optical line either using the filter or not, has been analyzed. The insertion loss of the cable with or without red filter is visualized in Fig. 15, also with it logarithm and linear function of the data. 


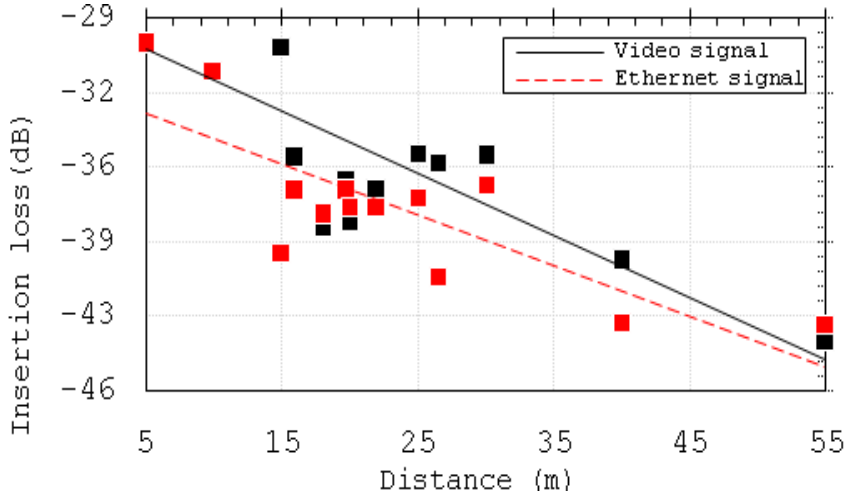

Fig. 13. Power loss comparison between green and red LED (and in linear function), green LED represent the video quality of the system while red LED represent the rate of download and upload through internet line

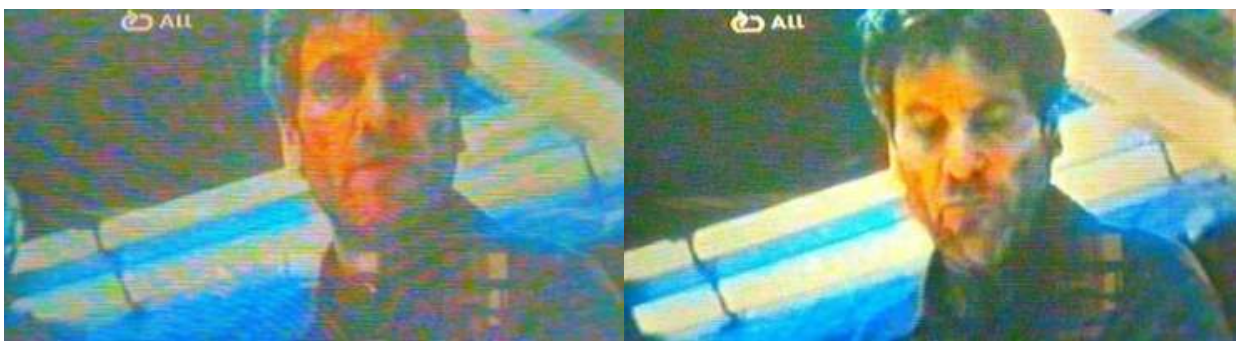

(a)

(b)

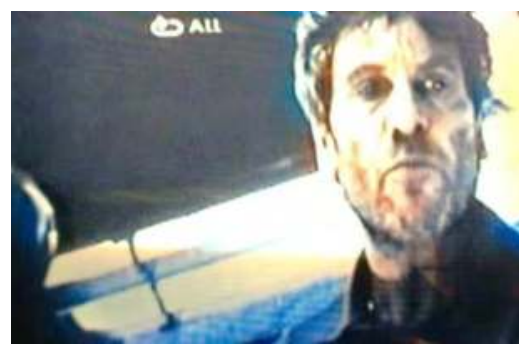

(c)

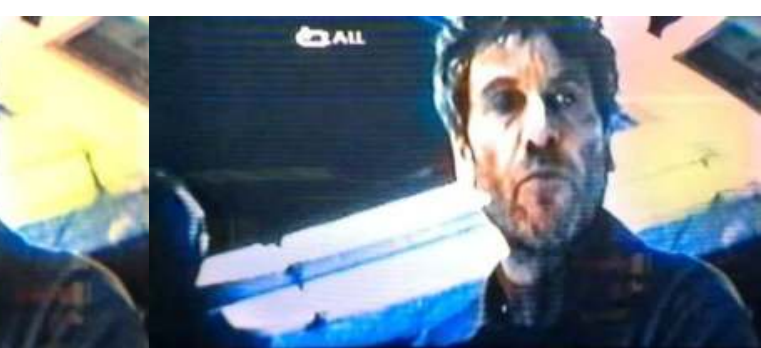

(d)

Fig. 14. Video quality of WDM-POF system of (a) 50m, (b) 30m, (c) 20m and (D) $10 \mathrm{~m}$ of optical transmission line 


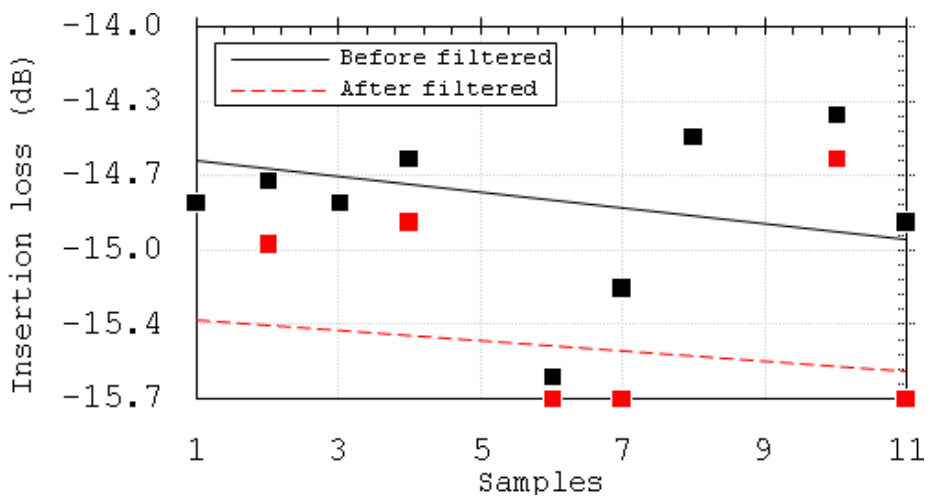

Fig. 15. Insertion loss of the cable (in logarithm function) before and after we place the red filter.

Figure 15 shows that almost $0.5 \mathrm{~dB}$ breakdown occurred once we placed a red filter into the line. But this deviation is not really influenced either speed rate on LAN network or the video quality which is displayed in monitor. Hence, it is shown that the deviation of insertion loss is about less then $-3 \mathrm{~dB}$ and the highest is reach $-7 \mathrm{~dB}$.

\section{Applications}

\subsection{In-vehicle entertainment networking}

The optical splitter is then applied in the automotive test bed to develop in-car infotainment. Two LEDs are used to perform intensity modulation with the internet data (red) and video signal (green) respectively. Our splitter has going advancement to function as demultiplexer to enable the multiplexed LED light been separated and interpreted next. As the result, the capacity of the communication become has double (due to use of two LEDs) and more application can be embedded in the system. Fig. 16 shows the result of LED WDM communication in the in-car application. The small diagram below shows the LED WDM communication configuration. Our proposed system is the lowest cost of WDM system that is reported up to this time. 

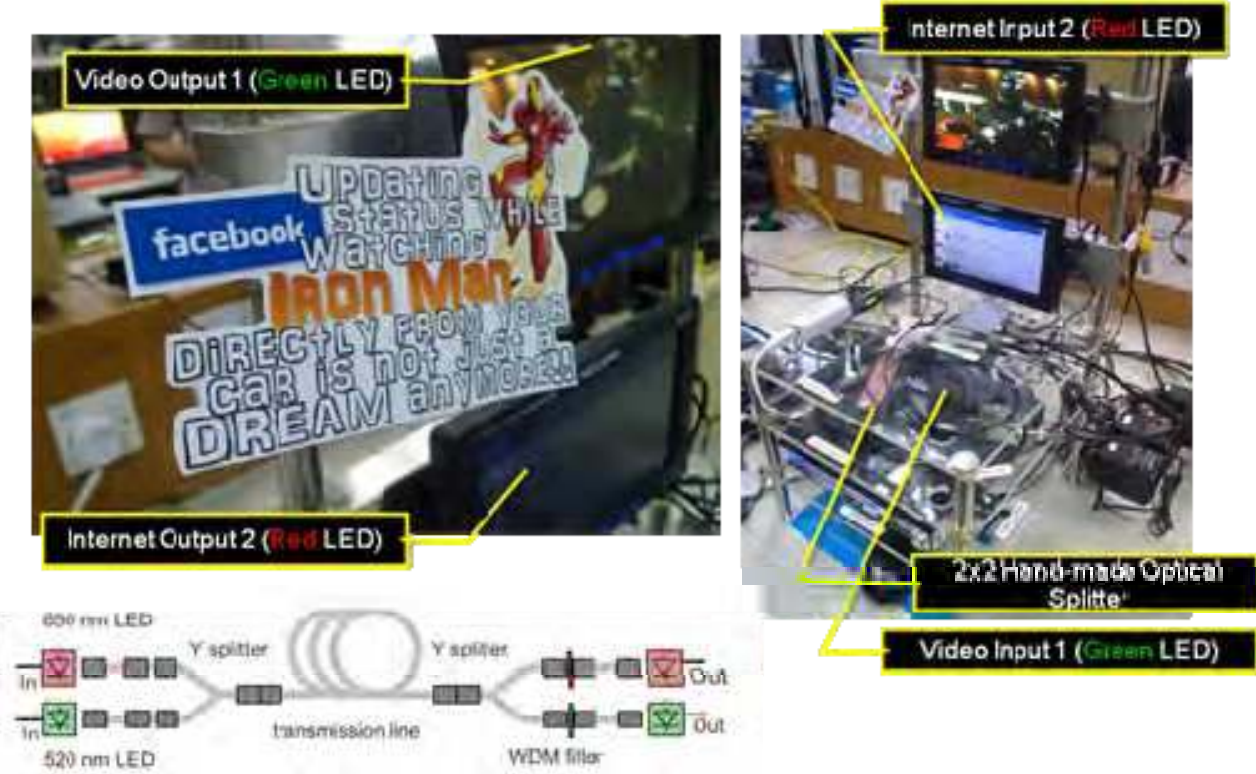

Video Input 1 (Giean LED)

Fig. 16. GT-POF splitter and demultiplexer play an important role in WDM communication in-vehicle entertainment networking (protected by patent no. PI2010700001)

\subsection{Low cost video signal splitting solution}

Closed-circuit television (CCTV) is not a cheap and simple system. The most powerful it is the more money we spend to built the overall system. But now, with a novel POF-based device, video signal splitting process will never be face pricing constraints anymore. So here, we can see that this device able to become the next video signal splitting solution (see Fig. 17). While some other CCTV and network supplier competing each other to provide the most powerful for surveillance system which come out with an expensive budget, now with combination between GT-POF splitter and an inexpensive GT-WDM-POF network, price will never be a main problem to install CCTV system anymore. 

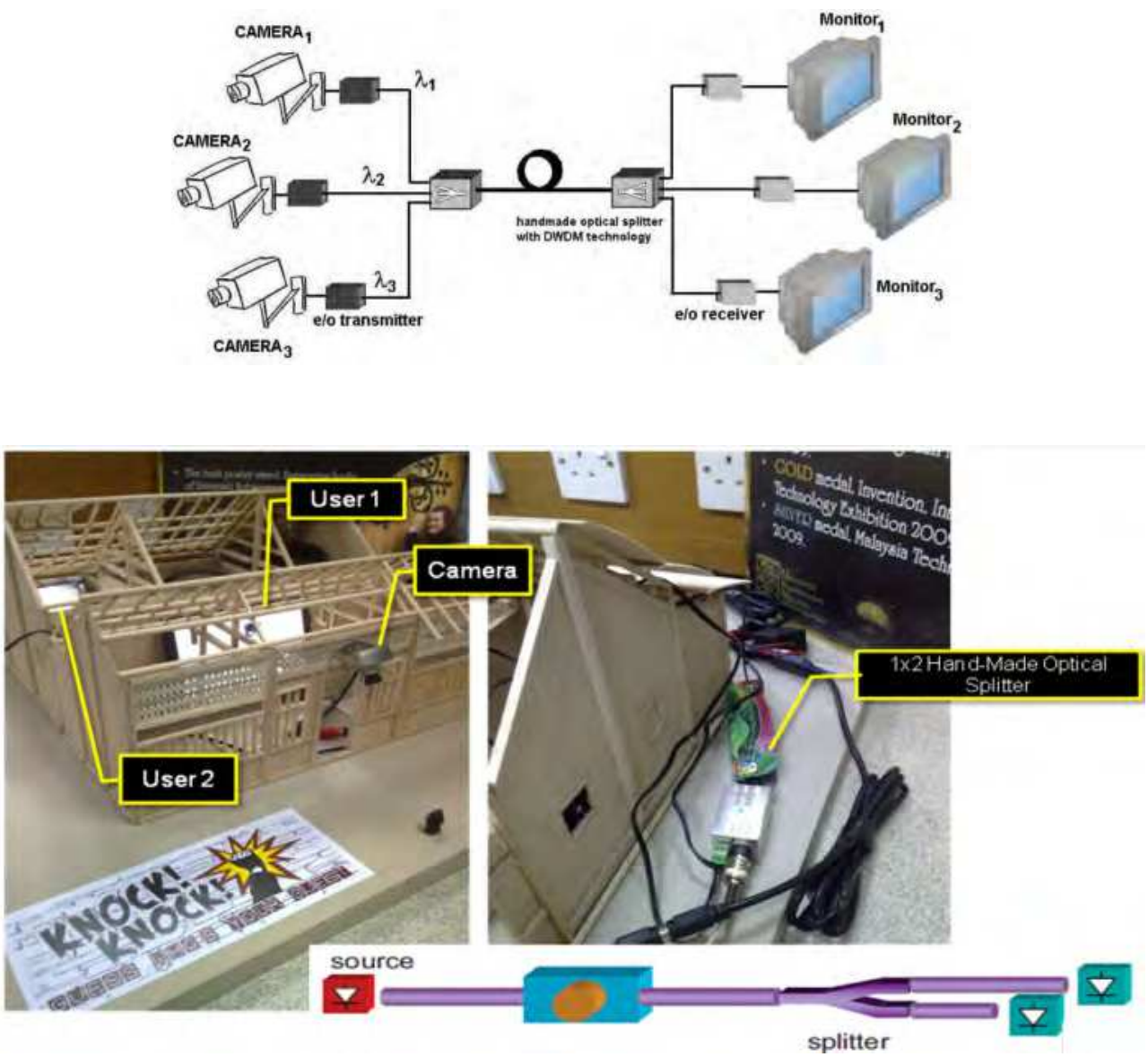

Fig. 17. Application for home networking and surveillance system in-house (protected by patent no. PI2010700001)

'Knock.. knock.. whose guest stands in front of the main door?', is one of the most powerful campaign regarding to this surveillance camera system. We can place a mini video camera in front of our main door of the house. Connecting the camera with some monitors display which is placed into a number of rooms inside the house. While we connect into some nods using a GT-POF splitter to split a video signal into a number of rooms, using a simple image processing devices, image from the main door will be transmitted into the house. Now, the members of a family inside the house, will never fighting anymore, ask one to another to open the main door while all of the members are busy with their own activity inside their own room. Because now, GT-POF splitter able to transmit an image from the main door into the rooms, and they will accurately noticed whose guest is standing in front of the door. 


\section{Conclusion}

Fusion technique has been successfully practiced to fabricate optical $1 \times N$ GT-POF splitter. Which $N$ represents numbers of output channel have been fabricated. Final analysis shows that efficiency of $1 \times 4$ GT-POF splitter output has splitting ratio 25:16:31:28 \% and $2.41 \mathrm{~dB}$ of minimum optical loss. Hence, the obtained result reveals that a novel $1 \times N$ GT-POF splitter has great potential to be employed as economical wavelength divisions multiplexer because it able to couple several different wavelengths with few main advantages that are low optical loss and low-cost. An intensive study suggested in order improving the homogeneity of this prototype. This device is highly recommended for GT-WDM-POF system as it is not as costly as other commercial POF splitter. Filters play an important role in giving a higher insertion loss from the GT-WDM-POF system, but the quality of a number of output ports is not badly destructed due to the color band gap from the filter itself, speed rate of the internet still stable and the resolution of the video image is quite good.

\section{Acknowledgment}

This research has been conducted in Computer\& Network Security Laboratory, Universiti Kebangsaan Malaysia (UKM). This project is supported by Ministry of Science, technology and Environment, Government of Malaysia, 01-01-02-SF0493 and Research University Grant fund UKM-GUP-TMK-07-02-108. All of our novel fabrication method of POF splitter, 1 Malaysia ${ }^{T M}$ splitter, $1 \times N$ GT-POF splitter and also the GT-WDM-POF network solution were protected by patent numbered PI2010700001.

\section{References}

Ab-Rahman, M. S., H. Guna, et al. (2009). 1xN Self-Made Polymer Optical Fiber Based Splitter for POF-650nm-LED based Application. 2009 International Conference on Electrical Engineering and Informatics, Selangor, Malaysia.

Ab-Rahman, M. S., H. Guna, et al. (2009). "Cost-effective 1x12 POF-Based Optical Splitters as an Alternative Optical Transmission Media for Multi-Purpose Application." IJCSNS International Journal of Computer Science and Network Security 9(3): 7278.

Ab-Rahman, M. S., H. Guna, et al. (2009). "Fabrication and Characterization of Optical 1x12 Fused-Taper-Twisted Polymer Optical Fiber Splitters." Journal of Optical Communications 30(1): 16-19.

Ab-Rahman, M. S., H. Guna, et al. (2008). "Fabrication and Characterization of CustomerMade 1x3 POFBased Optical Coupler for Home Networking." IJCSNS International Journal of Computer Science and Network Security 8(12): 43-48.

Ab-Rahman, M. S., H. Guna, et al. (2009). "Bidirectional Optical Power Measurement for High Performance Polymer Optical Fiber-based Splitter for Home Networking." Australian Journal of Basic and Applied Sciences 3(3): 1661-1669.

Ab-Rahman, M. S., M. H. Harun, et al. (2009). Comparative Analysis of Power Efficiency of Handmade 1×12 Polymer Optical Fiber-Based Optical Splitter. 2009 International Conference on Electrical Engineering and Informatics, Selangor, Malaysia. 
Appajaiah, A., V. Wachtendorf, et al. (2007). "Climatic exposure of polymer optical fibers: Thermooxidative stability characterization by chemiluminescence." Journal of Applied Polymer Science 103(3): 1593-1601.

Ehsan, A. A., S. Shaari, et al. (2009). Low Cost 1x2 Acrylic-based Plastic Optical Fiber Coupler with Hollow Taper Waveguide. 25TH of Progress in Electromagnetics Research Symposium, Beijing, China, Progress in Electromagnetics Research Symposium, PIERS.

Ericsson (2008) "Green Power to Bring Mobile Telephony to Billions of People."

Grzemba, A. (2008). MOST: the automotive multimedia network, Franzis.

Gupta, P. and H. Khurana (2010). Public Entrepreneurship: A Dynamic Strength For Budding Green Technology. Proceedings of the 4th National Conference; INDIACom-2010, New Delhi.

Held, G. (2002). Understandign Data Communications, Boston, EUA : Addison-Wesley.

Imoto, K., M. Maeda, et al. (1987). "New biconically tapered fiber star coupler fabricated by indirect heating method." Lightwave Technology, Journal of 5(5): 694-699.

Janota, A. and J. Hrbček (2011). Slovak ETC System Implemented - What Next? Transport Systems Telematics. J. Mikulski, Springer Berlin Heidelberg. 104: 30-37.

Keene, I. W. and R. K. Selli (1989). Passive components for plastic optical fibre transmission links. Plastics Materials for Optical Transmission, IEE Colloquium on.

Kelly, C., G. May, et al. (1995) "WDM Technologies in Telecommunications."

Kuzyk, M. (2007). Polymer fiber optics: materials, physics, and applications, CRC/Taylor \& Francis.

Schubert, E. F., J. K. Kim, et al. (2006). Solid-state lighting- : a benevolent technology. Bristol, ROYAUME-UNI, Institute of Physics.

Ziemann, O., P. Zamzow, et al. (2008). POF handbook: optical short range transmission systems, Springer. 


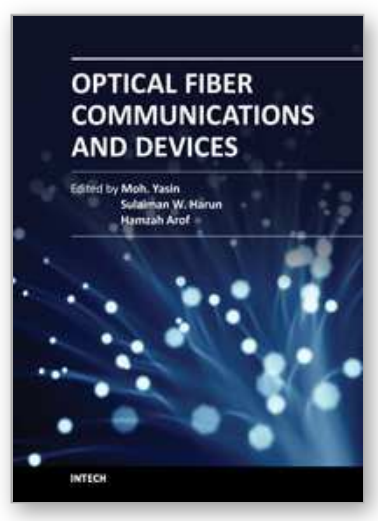

\author{
Optical Fiber Communications and Devices \\ Edited by Dr Moh. Yasin
}

ISBN 978-953-307-954-7

Hard cover, 380 pages

Publisher InTech

Published online 01, February, 2012

Published in print edition February, 2012

This book is a collection of works dealing with the important technologies and mathematical concepts behind today's optical fiber communications and devices. It features 17 selected topics such as architecture and topologies of optical networks, secure optical communication, PONs, LANs, and WANs and thus provides an overall view of current research trends and technology on these topics. The book compiles worldwide contributions from many prominent universities and research centers, bringing together leading academics and scientists in the field of photonics and optical communications. This compendium is an invaluable reference edited by three scientists with a wide knowledge of the field and the community. Researchers and practitioners working in photonics and optical communications will find this book a valuable resource.

\title{
How to reference
}

In order to correctly reference this scholarly work, feel free to copy and paste the following:

Mohammad Syuhaimi Ab-Rahman, Hadi Guna, Mohd Hazwan Harun, Latifah Supian and Kasmiran Jumari (2012). Integration of Eco-Friendly POF Based Splitter and Optical Filter for Low-Cost WDM Network Solutions, Optical Fiber Communications and Devices, Dr Moh. Yasin (Ed.), ISBN: 978-953-307-954-7, InTech, Available from: http://www.intechopen.com/books/optical-fiber-communications-and-devices/integration-of-ecofriendly-pof-based-splitter-and-optical-filter-for-low-cost-wdm-network-solutions

\section{INTECH}

open science | open minds

\author{
InTech Europe \\ University Campus STeP Ri \\ Slavka Krautzeka 83/A \\ 51000 Rijeka, Croatia \\ Phone: +385 (51) 770447 \\ Fax: +385 (51) 686166 \\ www.intechopen.com
}

\author{
InTech China \\ Unit 405, Office Block, Hotel Equatorial Shanghai \\ No.65, Yan An Road (West), Shanghai, 200040, China \\ 中国上海市延安西路65号上海国际贵都大饭店办公楼 405 单元 \\ Phone: +86-21-62489820 \\ Fax: +86-21-62489821
}


(C) 2012 The Author(s). Licensee IntechOpen. This is an open access article distributed under the terms of the Creative Commons Attribution 3.0 License, which permits unrestricted use, distribution, and reproduction in any medium, provided the original work is properly cited. 